\title{
Image denoising using TV-Stokes equation with an orientation-matching minimization *
}

\author{
Xue-Cheng Tai ${ }^{1,2}$, Sofia Borok ${ }^{1}$, and Jooyoung Hahn ${ }^{1}$ \\ 1 Division of Mathematical Sciences, School of Physical Mathematical Sciences, \\ Nanyang Technological University, Singapore. \\ 2 Mathematics Institute, University of Bergen, Norway, \\ xctai@ntu.edu.sg
}

\begin{abstract}
In this paper, we propose an orientation-matching minimization for denoising digital images with an additive noise. Inspired [1-3] by the two-step algorithm in the TV-Stokes denoising process, the regularized tangential vector field with the zero divergence condition is used in the first step. The present work suggests a different approach in order to reconstruct a denoised image in the second step. Namely, instead of finding an image that fits the regularized normal direction from the first step, we minimize an orientation between the image gradient and the regularized normal direction. It gives a nonlinear partial differential equation (PDE) for reconstructing denoised images, which has the diffusivity depending on an orientation of a regularized normal vector field and the weighted self-adaptive force term depending on the direction between the gradient of an image and the vector field. This allows to obtain a denoised image which has sharp edges and smooth regions, even though an original image has smoothly changing pixel values near sharp edges. The additive operator splitting scheme is used for discretizing Euler-Lagrange equations. We show improved qualities of results from various numerical experiments.
\end{abstract}

\section{Introduction}

Digital image denoising processes based on partial differential equations (PDEs) and energy minimization have been extensively studied for last 20 years in both theoretical and practical ways. From the Gaussian filtering to the anisotropic diffusion [4-6] and the total variation (TV) minimization $[7,8]$, a noisy image has been denoised from poorly estimated derivative information. The TV-filtering is very effective for piecewise constant images and the anisotropic diffusion is adjustable to flow-like images. However, both approaches are not suitable for an image which has smoothly changing pixel values near sharp edges.

Since qualities of denoised images are seriously dependent on estimated derivative information, it has been a crucial topic to regularize derivatives of an image [9], that is, an orientational information $[1,10-12]$. Inspired by [1-3], we

\footnotetext{
* The research is supported by MOE (Ministry of Education) Tier II project T207N2202 and IDM project NRF2007IDMIDM002-010. In addition, the support from SUG 20/07 is also gratefully acknowledged.
} 
also use a regularization of the tangent vector field of an image with the zero divergence condition. The present work propose a different approach in order to reconstruct a denoised image from the regularized normal vector field, which we call an orientation-mathching minimization. That is, we minimize an orientation between the image gradient and the regularized normal direction. It gives a nonlinear PDE for reconstructing denoised images, which has the diffusivity depending on an orientation of the regularized normal vector field and the weighted self-adaptive force term depending on the direction between the gradient of an image and the vector field. This allows to obtain a denoised image which has sharp edges and smooth regions, even though an original image has smoothly changing pixel values near sharp edges.

The paper is organized as follows. In Section 2, we introduce the proposed model with a review of TV-Stokes (TVS) denoising algorithm [1,2]. Some numerical aspects are explained in Section 3. Several numerical examples are shown and different models are compared in Section 4. The paper is concluded in Section 5.

\section{Two-step denoising model}

\subsection{Review of TV-Stokes denoising algorithm}

Let us consider a gray true image $d: \Omega \subset \mathbf{R}^{2} \rightarrow[0,1]$. We assume that a given noisy image $d_{0}$ has an additive Gaussian white noise $\eta$ with the relation

$$
d_{0}(\mathbf{p})=d(\mathbf{p})+\eta(\mathbf{p}), \quad \mathbf{p}=(x, y) \in \Omega .
$$

The normal and tangential vectors of the level curves of an image $d$ are given by

$$
\mathbf{n}=\nabla d(\mathbf{p})=\left(\frac{\partial d}{\partial x}, \frac{\partial d}{\partial y}\right)^{\mathrm{T}} \quad \text { and } \quad \mathbf{t}=\nabla^{\perp} d(\mathbf{p})=\left(\frac{\partial d}{\partial y},-\frac{\partial d}{\partial x}\right)^{\mathrm{T}},
$$

where $\mathrm{T}$ is a transpose. Then, the vector fields are satisfied with the following conditions

$$
\nabla \times \mathbf{n}=0 \quad \text { and } \quad \nabla \cdot \mathbf{t}=0,
$$

which means $\mathbf{n}$ is the irrotational vector field and $\mathbf{t}$ is the incompressible vector field. This property is very crucial when an image is reconstructed from the information of $\mathbf{n}$ or $\mathbf{t}$.

The TVS denoising model [1] consists of two steps to obtain a denoised image, which uses the same process in the second step as the Lysaker-OsherTai (LOT) model [10]. However, for the first step, instead of regularizing the normal vector field in the LOT model, a tangential vector field is regularized with the constraint of incompressibility. The regularized tangential vector field $\mathbf{t}$ is obtained by minimizing a functional:

$$
\min _{\nabla \cdot \mathbf{t}=0} \int_{\Omega}\left(|\nabla \mathbf{t}|+\frac{\delta}{2}\left|\mathbf{t}-\mathbf{t}_{0}\right|^{2}\right) d \mathbf{p}
$$


where $\mathbf{t}_{0}=\nabla^{\perp} d_{0}, \delta$ is a positive parameter, and $|\nabla \mathbf{t}|$ is defined by

$$
|\nabla \mathbf{t}|=\sqrt{\left(\frac{\partial u}{\partial x}\right)^{2}+\left(\frac{\partial u}{\partial y}\right)^{2}+\left(\frac{\partial v}{\partial x}\right)^{2}+\left(\frac{\partial v}{\partial y}\right)^{2}}, \nabla \mathbf{t}=\left(\begin{array}{c}
\nabla u \\
\nabla v
\end{array}\right), \mathbf{t}=\left(\begin{array}{c}
u \\
v
\end{array}\right) .
$$

The minimization problem is originally introduced in $[1,2]$. The optimality condition for the saddle point is obtained by the gradient descent flow which gives the PDE

$$
\begin{aligned}
\frac{\partial \mathbf{t}}{\partial \tau}-\nabla \cdot\left(\frac{\nabla \mathbf{t}}{|\nabla \mathbf{t}|}\right)+\delta\left(\mathbf{t}-\mathbf{t}_{0}\right)-\nabla \lambda & =0, \\
\nabla \cdot \mathbf{t} & =0,
\end{aligned}
$$

with the boundary conditions and the initial condition

$$
\left(\frac{\nabla \mathbf{t}}{|\nabla \mathbf{t}|}+\lambda I\right) \cdot \nu=0, \quad \mathbf{t}(\mathbf{p}, 0)=\mathbf{t}_{0},
$$

where $I$ is the identity matrix. Note that it is not straightforward to use the Perona-Malik (PM) model [4] or Rudin-Osher-Fatemi (ROF) model [7] directly for regularizing derivative information of an image [9]. One of reason for regularizing the tangential vector field is that the incompressibility condition, $\nabla \cdot \mathbf{t}=0$, is numerically computed using the Chorin projection type method which is well developed in the fluid dynamics; see details in Section 3. Moreover, the condition guarantees the existence of an image $d$ which satisfies the relation (2).

Once the regularized tangent vector field $\mathbf{t}=(u, v)^{\mathrm{T}}$ is obtained in the first step, the regularized normal vector field $\mathbf{n}$ is defined by $(v,-u)^{\mathrm{T}}$. In two-step algorithms for image denoising $[1,10]$ and image inpainting [2], it is suggested to solve the following minimization problem in the second step to reconstruct an image from $\mathbf{n}$ :

$$
\min _{\left\|d-d_{0}\right\|_{2}=\sigma} \int_{\Omega}\left(|\nabla d|-\nabla d \cdot \frac{\mathbf{n}}{|\mathbf{n}|}\right) d \mathbf{p},
$$

where $\|\cdot\|_{2}$ is the $L^{2}(\Omega)$ norm and $\sigma$ is the standard deviation of a Gaussian white noise. From the Euler-Lagrange equation and the gradient descent method along fictitious time $\tau$, we obtain a PDE for reconstructing an image with the free flux boundary condition and an initial condition $d(\mathbf{p}, 0)=d_{0}(\mathbf{p})$ :

$$
\frac{\partial d}{\partial \tau}(\mathbf{p}, \tau)=\nabla \cdot\left(\frac{\nabla d}{|\nabla d|}-\frac{\mathbf{n}}{|\mathbf{n}|}\right)-\mu\left(d-d_{0}\right),
$$

where $\mu$ is a positive parameter. Note that the ROF model is in the case of $\mathbf{n}=0$, which means that TV-norm filter is very suitable for denoising a piecewise constant image. In other words, the model suffers from a stair-case effect on regions whose pixel values are smoothly changed. Since the TVS denoising model and the LOT model find an image that fits the regularized normal vector field 
from the PDE (6), it is natural to have a better performance than the ROF model. However, it still has problems when the original image has smoothly changing pixel values near sharp edges and the regularized normal vector field on some regions is almost parallel or has some numerical errors; see Figures 2 and 4 .

\subsection{Orientation-matching minimization}

Inspired by the two-step algorithm in the TVS denoising model, we also use the regularized tangential vector field with the zero divergence condition in the first step. In this paper, we propose a new approach for reconstructing a denoised image in the second step. Namely, unlike finding an image that fits the regularized normal direction (5), we minimize an orientation between the image gradient and the regularized normal direction:

$$
\min _{\left\|d-d_{0}\right\|_{2}=\sigma} \int_{\Omega}\left(-\frac{|\nabla d \cdot \mathbf{n}|}{|\nabla d||\mathbf{n}|}\right) d \mathbf{p}
$$

where $\|\cdot\|_{2}$ and $\sigma$ are same in (5). From the Euler-Lagrange equation and the gradient descent method along fictitious time $\tau$, we obtain new PDE for obtaining a denoised image with the free flux boundary condition and an initial condition $d(\mathbf{p}, 0)=d_{0}(\mathbf{p})$ :

$$
\frac{\partial d}{\partial \tau}(\mathbf{p}, \tau)=\nabla \cdot\left(\frac{|\nabla d \cdot \mathbf{n}|}{|\nabla d|^{2}|\mathbf{n}|} \frac{\nabla d}{|\nabla d|}-\frac{\operatorname{sgn}(\nabla d \cdot \mathbf{n})}{|\nabla d|} \frac{\mathbf{n}}{|\mathbf{n}|}\right)-\mu\left(d-d_{0}\right),
$$

where $\operatorname{sgn}(\cdot)$ is the sign function and $\mu$ is a positive parameter. Unlike the diffusivity term $\frac{1}{|\nabla d|}$ and the fixed force $\nabla \cdot \frac{\mathbf{n}}{\mid \mathbf{n}}$ term in (6), the PDE from the proposed minimization has the diffusivity depending on an orientation of the regularized normal vector field $\mathbf{n}$ and the weighted self-adaptive force term depending on the direction between $\nabla d$ and $\mathbf{n}$.

We expect two differences between the proposed model (7) and the previous one (5) for reconstructing a denoised image. The first is that we have smaller orientation difference between the gradient of an original image and the gradient of a denoised image. The second is that the result in our model will have sharper edges in a denoised image, specially when the original image has smoothly changing pixel values near sharp edges. These are easily observed in numerical experiments and there are some plausible reasons.

In order to see the first difference, we assume that $\theta$ is the angle between $\nabla d /|\nabla d|$ and $\mathbf{n} /|\mathbf{n}|$. Then, the functional in the proposed model is written by

$$
\int_{\Omega}(-|\cos \theta|) d \mathbf{p} \text {. }
$$

and the functional in the previous model is presented by

$$
\begin{aligned}
\int_{\Omega}\left(|\nabla d|-\nabla d \cdot \frac{\mathbf{n}}{|\mathbf{n}|}\right) d \mathbf{p} & =\int_{\Omega}|\nabla d|\left(1-\frac{\nabla d \cdot \mathbf{n}}{|\nabla d||\mathbf{n}|}\right) d \mathbf{p} \\
& =\int_{\Omega}|\nabla d|(1-\cos \theta) d \mathbf{p} .
\end{aligned}
$$


The previous energy functional minimizes both $|\nabla d|$ and the angle $\theta$. If an image $d$ has some regions where $|\nabla d|$ is large enough, the minimization of the angle difference between $\nabla d /|\nabla d|$ and $\mathbf{n} /|\mathbf{n}|$ has quite an weak effect. In case of very small $|\nabla d|$, any angle will fit to $\mathbf{n} /|\mathbf{n}|$. Even though there exists a small amount of the angle difference, the graph of a denoised image is easily affected to generate a different shape to the original image. Since the proposed energy functional only minimizes the orientation difference, the shape of a denoised result is more sensitively changed in order to fit the original image regardless of the magnitude of $|\nabla d|$. We numerically show the orientation difference in Table 1 using different methods.

When we assume that $\nabla d$ is approximately parallel to $\mathbf{n}$, the second difference is expected because the proposed PDE can be written by

$$
\nabla \cdot\left(\frac{|\nabla d \cdot \mathbf{n}|}{|\nabla d|^{2}|\mathbf{n}|} \frac{\nabla d}{|\nabla d|}-\frac{\operatorname{sgn}(\nabla d \cdot \mathbf{n})}{|\nabla d|} \frac{\mathbf{n}}{|\mathbf{n}|}\right) \simeq \nabla \cdot\left(\left(\frac{\nabla d}{|\nabla d|}-( \pm) \frac{\mathbf{n}}{|\mathbf{n}|}\right) \frac{1}{|\nabla d|}\right) .
$$

From the approximation, if $|\nabla d|$ is large, we observe that the proposed model (8) is dominantly influenced by a data fidelity term and slightly affected by a regularization term. However, the previous model (6) is still affected by an additional force term from the regularized normal vector field. Since we may have some numerical errors of the vector field in a numerical computation of (3), it is difficult to know whether the additional force will generate a good result or not. Even though the extra force reduces a stair-case effect comparing to the TV-filtering method in smooth regions, it may derive an erroneous effect near edges where $|\nabla d|$ is large. We numerically show qualities of a denoised image when the original image has smoothly changing pixel values near sharp edges; see Figure 2, 3, and 4 .

\section{Numerical Aspects}

For the discretization, we use the standard staggered grid which is suggested in [2]. In this section, we briefly note some issues of discretization in the first and second steps.

\subsection{A regularization of the tangent vector field}

The minimization problem (3) for regularizing the tangent vector filed with the constraint of the incompressibility condition is solved by the method of Lagrange and the Chorin projection type method. We apply the Chorin projection type method and the AOS method $[13,14]$ to solve the PDE (4).

1. Calculation for an intermediate tangent field $\mathbf{t}^{*}$ which is not incompressible vector field.

$$
\frac{\mathbf{t}^{*}-\mathbf{t}^{n}}{\Delta \tau}=\nabla \cdot\left(\frac{\nabla \mathbf{t}^{*}}{\left|\nabla \mathbf{t}^{n}\right|_{\epsilon}}\right)-\delta\left(\mathbf{t}^{*}-\mathbf{t}_{0}\right)
$$


with the boundary condition

$$
\nabla \mathbf{t}^{*} \cdot \nu=0
$$

where $\left|\nabla \mathbf{t}^{n}\right|_{\epsilon} \equiv \sqrt{\epsilon+\left|\nabla \mathbf{t}^{n}\right|^{2}}$ and $\mathbf{t}^{n}$ is the tangent vector field at the $n^{\text {th }}$ time step. The AOS method of the linearized equation for the component $u$ and $v$ is used. The spatial derivatives with respect to $x$ and $y$ are approximated by standard one-sided finite differences.

2. Solving for $\lambda$ such that

$$
\begin{cases}\frac{\mathbf{t}^{n+1}-\mathbf{t}^{*}}{\Delta \tau} & =\nabla \lambda \\ \nabla \cdot \mathbf{t}^{n+1} & =0\end{cases}
$$

This gives a Poisson equation for $\lambda$ with the zero Neumann boundary condition:

$$
\nabla \cdot \nabla \lambda=-\frac{1}{\Delta \tau} \nabla \cdot \mathbf{t}^{*} .
$$

3. Updating the tangent vector field by

$$
\mathbf{t}^{n+1}=\mathbf{t}^{*}+\Delta \tau \nabla \lambda .
$$

The boundary values are updated by the incompressibility condition. More datails are shown in [1].

For the stopping criterion, we use the steady state condition for the flow $\mathbf{t}=(u, v)^{\mathrm{T}}$ :

$$
\max \left(\frac{\left\|u^{n+1}-u^{n}\right\|_{\infty}}{\left\|u^{n}\right\|_{\infty}}, \frac{\left\|v^{n+1}-v^{n}\right\|_{\infty}}{\left\|v^{n}\right\|_{\infty}}\right) \leq \alpha,
$$

where $n$ and $n+1$ are consecutive time steps and $\|\cdot\|_{\infty}$ is the $L^{\infty}(\Omega)$ norm. Note that $\alpha=10^{-4}$ is fixed for all examples in the paper.

\subsection{A reconstruction of a denoised image}

After the regularized tangent vector field $\mathbf{t}=(u, v)^{\mathrm{T}}$ is computed from the first step, we propose an orientation-matching minimization (7) to reconstruct a denoised image from the regularized normal vector field $\mathbf{n}=(v,-u)^{\mathrm{T}}$. The optimality condition for the saddle point is obtained by the gradient descent flow which gives a PDE (8). We also apply the AOS method to solve the proposed PDE. Note that we use a regularized sign function:

$$
\operatorname{sgn}_{\varepsilon}(s) \equiv 2 H_{\varepsilon}(s)-1, \quad H_{\varepsilon}(s) \equiv \begin{cases}1 & s>\varepsilon \\ 0 & s<\varepsilon \\ \frac{1}{2}\left(1+\frac{s}{\varepsilon}+\frac{1}{\pi} \sin \left(\frac{\pi s}{\varepsilon}\right)\right) & \text { otherwise }\end{cases}
$$


and a parameter $\epsilon$ is used to avoid division by zero in numerical experiments:

$$
\left|\nabla d^{n}\right|_{\epsilon} \equiv \sqrt{\epsilon+\left|\nabla d^{n}\right|^{2}}, \quad|\mathbf{n}|_{\epsilon} \equiv \sqrt{\epsilon+|\mathbf{n}|^{2}},
$$

where $n$ is the $n^{\text {th }}$ time step. More datails are shown in [1].

For the stopping criterion, we use the steady state condition for the relative difference in the energy (7). That is,

$$
\frac{\left|E^{n+1}-E^{n}\right|}{E^{n}} \leq \beta,
$$

where $E^{n}$ is the energy value at the time step $n$ approximated by

$$
E^{n} \approx \sum_{i, j}\left(-\frac{\left|\nabla d^{n} \cdot \mathbf{n}\right|}{\left|\nabla d^{n}\right|_{\epsilon}|\mathbf{n}|_{\epsilon}}\right) .
$$

The value of $\beta$ may be different for images and we use $10^{-2} \leq \beta \leq 10^{-4}$. The energy (5) is similarly computed and it is used for the stopping criterion of the second step in the previous model.

Remark 1. The right choice of parameters is crucial for qualities of a denoised image. The parameters, $\delta$ and $\mu$, they control a balance between a data smoothing and a fidelity therm. The parameter $\epsilon$ is used to avoid a division by zero, which also controls the diffusivity for smoothing a data. The AOS scheme provides us a wide range of the time step. However, if $\Delta \tau$ is too large, then visual qualities of a denoised image are deteriorated.

\section{Examples}

In this section, we show numerical experiments for denoising an image based on the proposed method. With synthetic images and real images, we discuss about the strength of the proposed orientation-matching minimization and compare with results from other methods. For the simplicity, the following notations are used to indicate parameters in different methods.

- $V(\Delta \tau, \delta, \epsilon)$ : a regularization of the tangent vector field (4).

- $M^{1}(\Delta \tau, \mu, \epsilon)$ : a reconstruction of a denoised image from (8).

- $M^{2}(\Delta \tau, \mu, \epsilon)$ : a reconstruction of a denoised image from (6).

- $M^{3}(\lambda)$ : the TV-filtering method in [8].

- $M^{4}(\mu, \rho, \epsilon)$ : a reconstruction of a denoised image from (11).

We also include an interesting numerical experiment to combine the anisotropic nonlinear diffusion $[5,6]$ with the regularized tangent vector field $\mathbf{t}=(u, v)^{\mathrm{T}}$ in the first step (3). That is, the diffusivity tensor is constructed from $\mathbf{n}=(v,-u)^{\mathrm{T}}$ and we solve a PDE with the free flux boundary condition:

$$
\frac{\partial d}{\partial \tau}(\mathbf{p}, \tau)=\nabla \cdot\left(g\left(G_{\rho} * \mathbf{n n}^{\mathrm{T}}\right) \nabla d\right)-\mu\left(d-d_{0}\right),
$$



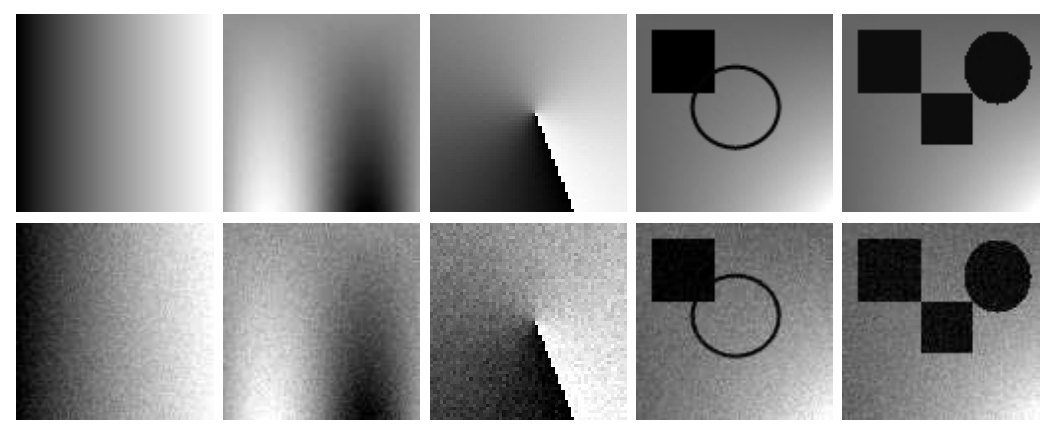

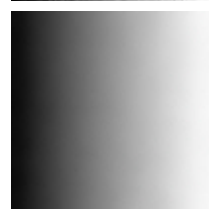

(test 1)

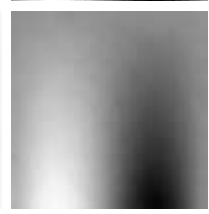

(test 2)

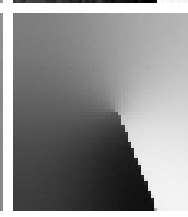

(test 3)

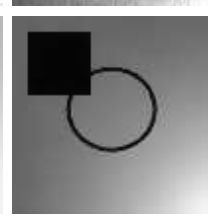

(test 4)

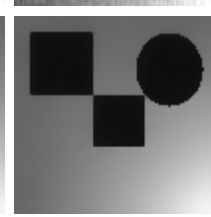

(test 5)

Fig. 1. Results from the proposed method: the first row is original images, we add a Gaussian white noise with zero mean and the standard deviation 10 for all images in the second row, and the last row is the result from the proposed method.

where $\left(G_{\rho} * M\right)_{i j}=G_{\rho} * m_{i j}$ for a matrix $M=\left(m_{i j}\right)$ and $G_{\rho} * f$ is the convolution of $f$ with the two-dimensional Gaussian kernel with the standard deviation $\rho$. The function $g$ is defined on a set $\mathcal{S}$ of real semi-positive symmetric $2 \times 2$ matrices:

$$
g(M) \equiv \frac{1}{\sqrt{\epsilon+\Lambda_{1}}} v_{\Lambda_{1}} v_{\Lambda_{1}}{ }^{\mathrm{T}}+\frac{1}{\sqrt{\epsilon+\Lambda_{2}}}\left(\Lambda_{2}\right) v_{\Lambda_{2}} v_{\Lambda_{2}}{ }^{\mathrm{T}}
$$

where $\left(\Lambda_{1}, v_{\Lambda_{1}}\right)$ and $\left(\Lambda_{2}, v_{\Lambda_{2}}\right)$ are eigenpairs of $M \in \mathcal{S}, \Lambda_{1} \geq \Lambda_{2}$.

Table 1. Comparison of the orientation difference $\gamma$ in (12): (A) is the result of the proposed method, (B) is the result of TVS denoising method, (C) is the result of TVfilter method. The denoised image from the prosed method is shown in the third row of Figure 1.

\begin{tabular}{cccc}
\hline images & $(\mathrm{A})$ & $(\mathrm{B})$ & $(\mathrm{C})$ \\
\hline test 1 & 0.9706 & 0.9316 & 0.7466 \\
test 2 & 0.8693 & 0.8478 & 0.6825 \\
test 3 & 0.7668 & 0.6304 & 0.6218 \\
test 4 & 0.5681 & 0.4983 & 0.3891 \\
test 5 & 0.4936 & 0.4051 & 0.3228 \\
\hline
\end{tabular}

Example 1. We numerically check how well the orientation of the gradient of a denoised image is fitted to the gradient of the original image. In Table 1, 


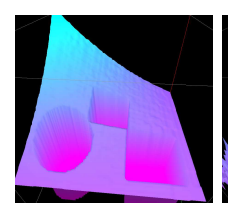

(a)

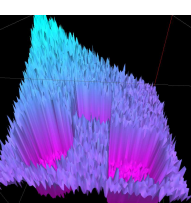

(b)

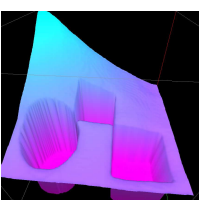

(c)

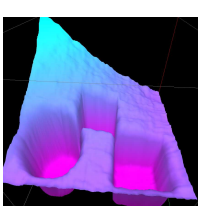

(d)

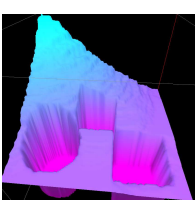

(e)

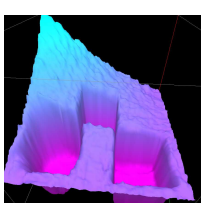

(f)

Fig. 2. Comparison with other methods: (a), (b), and (c) are the graph of images from top to bottom of the test 5 in Figure 1, respectively. (d) is the result of TVS denoising model and (e) is the result of TV-filtering model. (f) is the result from (11). The parameters are shown in Example 1. Note that (b) is the result from the proposed model.

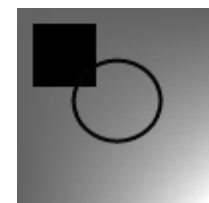

(a)

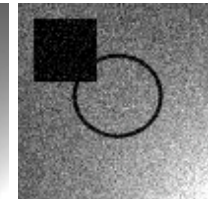

(b)

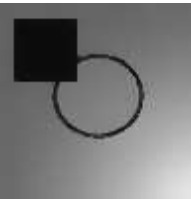

(c)

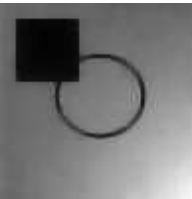

(d)

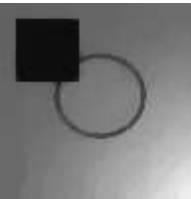

(e)

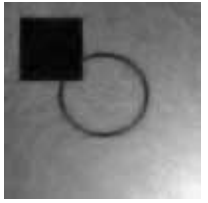

(f)

Fig. 3. (a) is an original image. We add a Gaussian white noise with zero mean and the standard deviation 20 in (b) which is larger noise than in test 4 in Figure 1. (c) is the result of the proposed model. (d) is the result of TVS denoising model and (e) is the result of TV-filtering model. (f) is the result from (11).

we measure the orientation difference for different test images:

$$
\gamma=\frac{1}{|\Omega|} \int_{\Omega}\left|\frac{\nabla d_{e}}{\left|\nabla d_{e}\right|} \cdot \frac{\nabla d_{c}}{\left|\nabla d_{c}\right|}\right| d \mathbf{p}
$$

where $d_{e}$ is the original image, $d_{c}$ is the computed denoised image, and $|\Omega|$ is the area of the domain. In the first step in (A) and (B), $V\left(10^{-1}, 1,10^{4}\right)$ is fixed for all test images. In the second step in (A) and (B), $M^{1}\left(10^{-3}, 1,10^{-3}\right)$ and $M^{2}\left(10^{-3}, 1,10^{-6}\right)$ for test $1, M^{1}\left(10^{-3}, 1,5 \cdot 10^{-3}\right)$ and $M^{2}\left(10^{-3}, 1,2.5 \times\right.$ $\left.10^{-5}\right)$ for test $2, M^{1}\left(10^{-3}, 1,2.5 \cdot 10^{-5}\right)$ and $M^{2}\left(10^{3}, 1,5 \cdot 10^{-3}\right)$ for test 3 , $M^{1}\left(10^{-3}, 1,10^{-3}\right)$ and $M^{2}\left(10^{-3}, 5,10^{-3}\right)$ for test 4 , and $M^{1}\left(10^{-3}, 2,3 \times 10^{-3}\right)$ and $M^{2}\left(10^{3}, 3,3 \times 10^{-3}\right)$ for test 5 are used, respectively. In (C), all results are obtained by $M^{3}(60)$. As we explain in Section 2.2, the proposed model has better performance for fitting the orientation. In Figure 2, the graph of computed results are presented in order to see visual difference. The result (f) is obtained by (11) with $M^{4}\left(0.4,0.1,10^{-3}\right)$. A denoised image from the proposed method has very clean shape, even though an original image has smoothly changing pixel values near edges. We observe that results from other methods do not have very sharp edges. The result (e) from the TV-filtering model has has a stair-case effect on smooth regions. These results are expected in Section 2.2. 


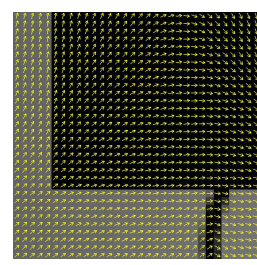

(a)

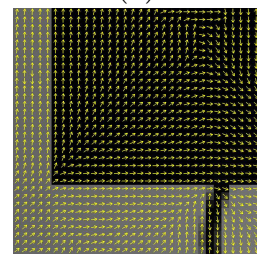

(b)

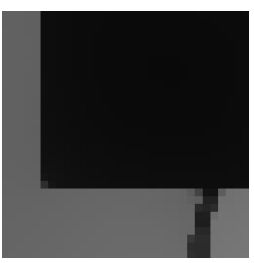

(a1)

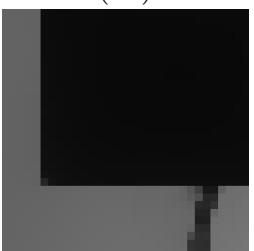

(b1)

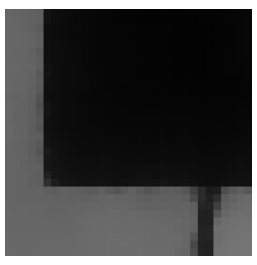

(a2)

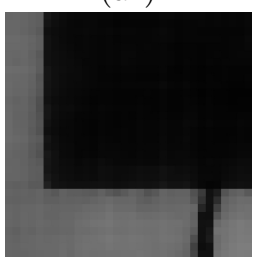

(b2)

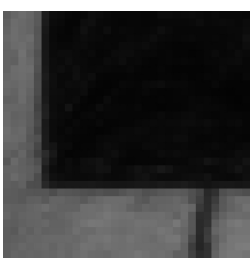

(a3)

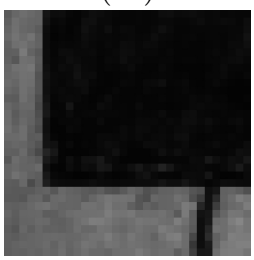

(b3)

Fig. 4. (a) is a part of a tangent vector field from (3). (a1), (a2), and (a3) in the first row are a part of the images (c), (d), (f) in Figure 3, respectively. In the second row, we compute less smooth tangent vector field (b) in the first step and use the same method for the second step as the first row.

Example 2. In Figure 3, we compare results from different methods with larger noise in Figure 1. For a regularization of the tangent vector field in (c) and (d), $V\left(5 \times 10^{-2}, 1,10^{-4}\right)$ is used. The result of the proposed method in (c) is obtained by $M^{1}\left(10^{-3}, 2,10^{-3}\right)$. (d), (e), and (f) are obtained by $M^{2}\left(10^{-3}, 4,10^{-4}\right)$, $M^{3}(80)$, and $M^{4}\left(0.5,1,10^{-3}\right)$. Now, we observe the effect of the first step (3) to the second step in (8), (6), and (11) is numerically shown. The first row in Figure 4 is a part of images in Figure 3. In the second row, we obtain a relatively less smooth vector field with $V\left(10^{-1}, 3,10^{-4}\right)$. (b2) is obtained by $M^{1}\left(10^{-3}, 2,10^{-3}\right.$ and we use same parameters for (b1) and (b3) as (a1) and (a3). Note that the result (b2) does not have very clean edge even if we use smaller $\mu$ in the second step for the previous model (6). The other methods, (6) and (11), are responded by a small change of the vector field because the field is directly used in the formulation without considering any relation with an image data.

Example 3. For real images, we compare with denoised images from different methods. In Figure 5, the image (a) is obtained by the proposed method with $V\left(10^{-1}, 5,10^{-4}\right)$ and $M^{1}\left(5 \times 10^{-4}, 5,5 \times 10^{-4}\right)$. (b) is from $V\left(5 \times 10^{-2}, 5,10^{-4}\right)$ and $M^{2}\left(10^{-3}, 1,5 \times 10^{-3}\right)$. (c) is from $M^{3}(60)$. In Figure 6 , the image (a) is obtained by the proposed method with $V\left(10^{-1}, 2,10^{-4}\right)$ and $M^{1}\left(10^{-4}, 30,10^{-3}\right)$. (b) is from $V\left(10^{-1}, 2,10^{-4}\right)$ and $M^{2}\left(10^{-3}, 2,10^{-3}\right)$. (c) is from $M^{3}(60)$. For these images, two models (5) and (7) give similar results which are better than the TV-filtering model. 


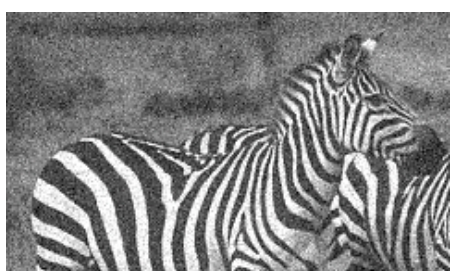

(a)

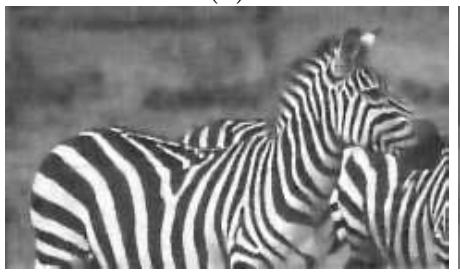

(c)

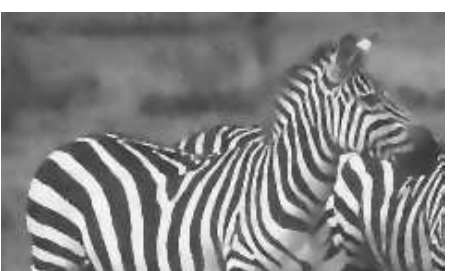

(b)

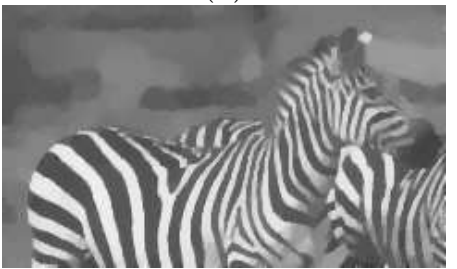

(d)

Fig. 5. There is a Gaussian white noise with zero mean and the standard deviation 10 in (a) from [15]. (b) is the result from the proposed model. (c) is the result of TVS denoising model and (d) is the result of TV-filtering model. The size of image is $240 \times 124$.

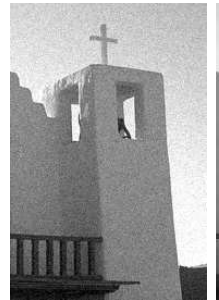

(a)

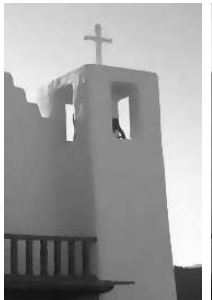

(b)

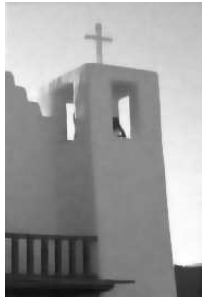

(c)

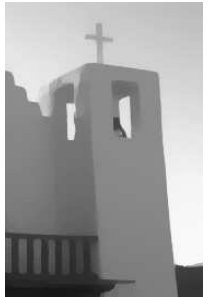

(d)

Fig. 6. There is a Gaussian white noise with zero mean and the standard deviation 10 in (a) from [15]. (b) is the result of the proposed model. (c) is the result of TVS denoising model and (d) is the result of TV-filtering model. The size of image is $181 \times 274$.

\section{Conclusions.}

We proposed an orientation-matching minimization for denoising digital images. Our algorithm consisted of two steps. In the first step, we use the regularized tangent vector field with the incompressibility condition which is suggested in [2]. The condition is crucial for reconstructing an image from the vector field. In the second step, the present work proposed a minimization of an orientation between the image gradient and the regularized normal direction. It gives a nonlinear PDE for reconstructing a denoised images, which has the diffusivity depending on an orientation of the regularized normal vector field and the weighted self-adaptive 
force term depending on the direction between the gradient of an image and the vector field. This allows to obtain a denoised image which has sharp edges and smooth regions, even though an original image has smoothly changing pixel values near sharp edges. We show improved qualities of results from various numerical experiments.

\section{References}

1. Rahman, T., Tai, X.C., Osher, S.: A TV-stokes denoising algorithm. In: Scale space and variational methods in Computer vision, F. Sgallari, A. Murli and N. Paragios eds, Springer, pp. 473-482. (2007)

2. Tai, X.C., Osher, S., Holm, R.: Image inpainting using tv-stokes equation. In: Image processing based on partial differential equations, Springer, Heidelberg (2006) 3-22

3. Bertalmio, M., and G. Sapiro, A.L.B.: Navier-Stokes, fluid dynamica, and image and video inpainting. In: Proc. Conf. Comp. Vision Pattern Rec. (2001) 355-362

4. Perona, P., Malik, J.: Scale space and edge detection using anisotropic diffusion. IEEE Trans. Pattern Anal. Machine Intell. 12(7) (1990) 629-639

5. Weickert, J.: Coherence-enhancing diffusion filtering. Int. J. Comput. Vis. 31 (1999) 111-127

6. Brox, T., Weickert, J., Burgeth, B., Mrázek, P.: Nonlinear structure tensors. Image Vis. Comput. 24 (2006) 41-55

7. Rudin, L.I., Osher, S., Fatemi, E.: Nonlinear total variation based noise removal algorithms. Physica D 60 (1992) 259-268

8. Bresson, X., Chan, T.: Fast daul minimization of the vectorial total variation norm and applications to color image processing. Inverse Problems and Imaging 2(4) (2008) 455-484

9. Hahn, J., Lee, C.O.: A nonlinear structure tensor with the diffusivity matrix composed of the image gradient. accepted to J. Math. Imag. Vis.

10. Lysaker, M., Osher, S., Tai, X.C.: Noise removal using smoothed normals and surface fitting. IEEE Trans. Image Processing 13(10) (2004) 1345-1357

11. Vese, L., Osher, S.: Numerical methods for p-harmonic flows and applications to image processing. SIAM J. Numer. Anal. 40(6) (2002) 2085-2104

12. Sochen, N., Sagiv, C., Kimmel, R.: Stereographic combing a porcupine or studies on direction diffusion in image processing. SIAM J. Appl. Math. 64(5) (2004) $1477-1508$

13. Lu, T., Neittaanmaki, P., Tai, X.C.: A parallel splitting up method for partial differential equations and its application to navier-stokes equations. RAIRO Math. Model. and Numer. Anal. 26(6) (1992) 673-708

14. Weickert, J., ter Harr Romeny, B.M., Viergever, M.A.: Efficient and reliable schemes for nonlinear diffusion filtering. IEEE Trans. Image Processing 7 (2001) 398-410

15. Martin, D., Fowlkes, C., Tal, D., Malik, J.: A database of human segmented natural images and its application to evaluating segmentation algorithms and measuring ecological statistics. In: Proc. 8th Int'l Conf. Computer Vision. Volume 2. (July 2001) 416-423 